\title{
Input/Output Analysis of Hypersonic Boundary Layers using the One-Way Navier-Stokes (OWNS) Equations
}

\author{
Omar Kamal* \\ California Institute of Technology, Pasadena, California, 91106, United States \\ Georgios Rigas ${ }^{\dagger}$ \\ Imperial College London, South Kensington, London, SW7 2AZ, United Kingdom \\ Matthew T. Lakebrink \\ The Boeing Company, Hazelwood, MO, 63042, United States \\ Tim Colonius $\S$ \\ California Institute of Technology, Pasadena, California, 91106, United States
}

\begin{abstract}
Accurate prediction of linear amplification of disturbances in hypersonic boundary layers is computationally challenging. While direct numerical simulations (DNS) and global analysis can be used to compute optimal (worst-case) disturbances and forced responses, their large computational expense render these tools less practical for large design parameter spaces. At the same time, parabolized stability equations can be unreliable for problems involving multimodal and non-modal interactions. To bridge this gap, we apply an approximate fast marching technique, the One-Way Navier-Stokes (OWNS) Equations, in iterative fashion to solve for optimal disturbances. OWNS approximates a rigorous parabolization of the equations of motion by removing disturbances with upstream group velocity using a higher-order recursive filter. Using OWNS, we aim to characterize disturbances of flat-plate hypersonic boundary layers over a range of Mach numbers, and find optimal disturbances under different cost functions that define corresponding receptivity problems. The calculation of optimal disturbances reveals multi-modal transition scenarios depending on the spatial support, frequency, and physical nature of the external disturbances.
\end{abstract}

\section{Nomenclature}

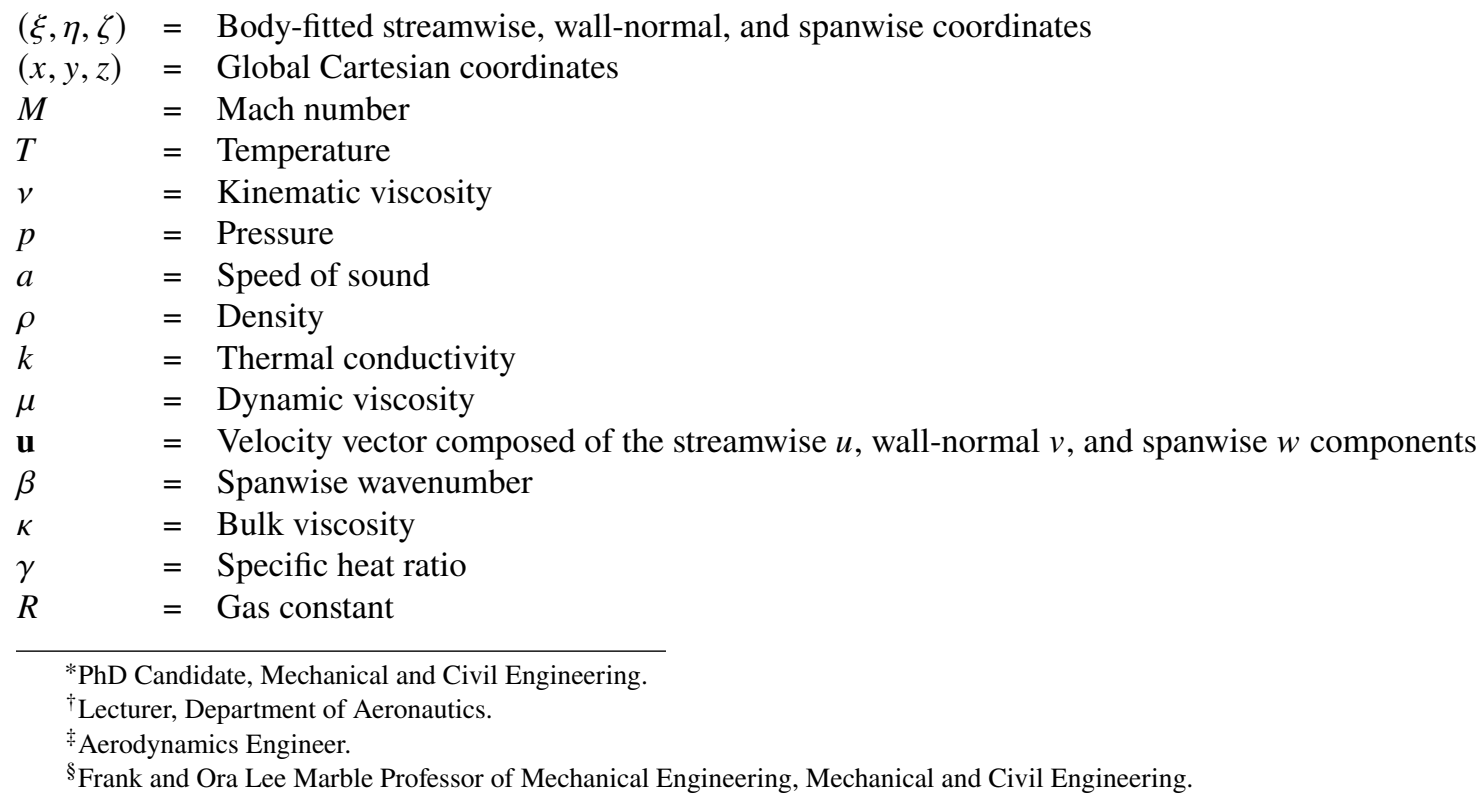




$\begin{array}{ll}\delta_{0}^{\dagger} & =\text { Inlet Blasius length scale defined as } \sqrt{\frac{v_{\infty}^{\dagger} \xi_{0}^{\dagger}}{u_{\infty}^{\dagger}}} \\ t & =\text { Time } \\ f & =\text { Disturbance frequency } \\ \omega & =\text { Angular disturbance frequency } \\ F & =\text { Non-dimensional frequency defined as } \frac{\omega^{\dagger} v_{\infty}^{\dagger}}{u_{\infty}^{\dagger}} \\ \Psi & =\text { Gain from optimization algorithms }\end{array}$

\section{Introduction}

Developing an in-depth understanding of hypersonic laminar-to-turbulent transition will help formulate methods to control aerodynamic and thermal loads. For example, due to the difficulty of predicting the onset of turbulence, the take-off mass of the US National Aerospace Plane (NASP/X-30) was affected by a factor of two or more [1]. Thus, to design an optimal vehicle, it is of utmost importance to understand the state of the aerodynamic boundary layer throughout the flight trajectory.

For idealized flow conditions, i.e. slender geometries experiencing minor pressure gradients and low freestream perturbations, at subsonic and low supersonic speeds, natural transition is due to the first mode, which is characterized by the amplification of Tollmien-Schlichting (T-S) waves [2]. In contrast, at hypersonic speeds $(M>4)$, natural transition is far more complex due to the presence and interaction of multiple discrete modes including the first, second, and higher-order Mack modes [3, 4]. These modes are characterized by convectively-amplified streamwise-propagating acoustic disturbances usually in the ultrasonic range, which approximately behave as acoustic waves reflecting between the solid wall and the relative sonic line [2]. The second Mack mode is usually the dominant instability in zero-pressuregradient boundary layers at hypersonic speeds with insulated walls. Additionally, it has been shown that the first mode along with other instabilities, such as the concave-wall Görtler instability [5], the supersonic mode [6], and three-dimensional crossflow instability [7, 8] interact with and/or modify the acoustic properties of the second mode (e.g. Görtler-modified azimuthal Mack-modes [9]). Thus, predictive tools which are capable of efficiently tracking these multi-modal instabilities are critical for engineering design.

Current tools to predict boundary-layer transition include direct numerical simulations (DNS) and its linear counterparts such as global stability analysis, linear stability theory (LST), and parabolized stability equations (PSE). Although global methods are most accurate, they are computationally intensive since they require full spatial discretization, especially with complex geometries. LST is more computationally efficient but typically employs the parallel-flow assumption that introduces error as seen in [10]. PSE may seem to be the optimal method between DNS and LST, but there are intrinsic disadvantages. Instead of formally deriving a one-way operator, PSE achieves a stable spatial march by numerically damping upstream-propagating waves, but this damping gives rise to unpredictable (uncontrolled) distortion of the downstream waves that one aims to resolve [11]. This has profound negative consequence for non-modal instabilities and for flows with more rapid streamwise evolution [11].

In traditional stability analyses, we analyze how incident disturbances evolve into flow instabilities. However, for design purposes, the inverse problem is more useful: what are the worst-case disturbances that lead to the fastest transition from laminar to turbulent flows. Recently, a new technique referred to as resolvent or input/output analysis has been introduced that combines the linear receptivity and instability problem via optimization techniques, such as singular value decomposition (SVD), to determine inflow disturbances that lead to maximal amplification [12]. These global analyses have been historically tractable for simple geometries at low-speeds [13], but their extension to high-speed flows have gained recent attention [14, 15]. While promising results have been shown, their application to practical, in-flight geometries would involve complex algorithms and large-scale computing.

We alleviate the aforementioned deficiencies by introducing a novel streamwise-marching technique, the One-Way Navier-Stokes (OWNS) equations. Originally developed for hyperbolic equations (e.g. Euler), OWNS employs a rigorous parabolization technique to generate well-posed, one-way approximations [16]. Efficient (fast) approximations of the resulting operator can then be made using recursive filters that were originally developed for non-reflecting boundary conditions (NRBC). Aside from the parabolization itself, and unlike PSE, the numerics are convergent and not restricted to a dominant wavelength. A schematic comparing the global, PSE, and OWNS method on a flat-plate boundary layer shown is shown in Fig. 11 We build on an earlier attempt [17, 18] to extend the OWNS algorithm to perform the inverse problem of determining the worst-case disturbances that give rise to the maximum achievable amplification (in a suitably defined metric). Unlike approaches that invoke the SVD of the global operator [12], 
the OWNS approach finds these solutions via an iterative, space-marching technique that appreciably reduces the computational burden without sacrificing accuracy.
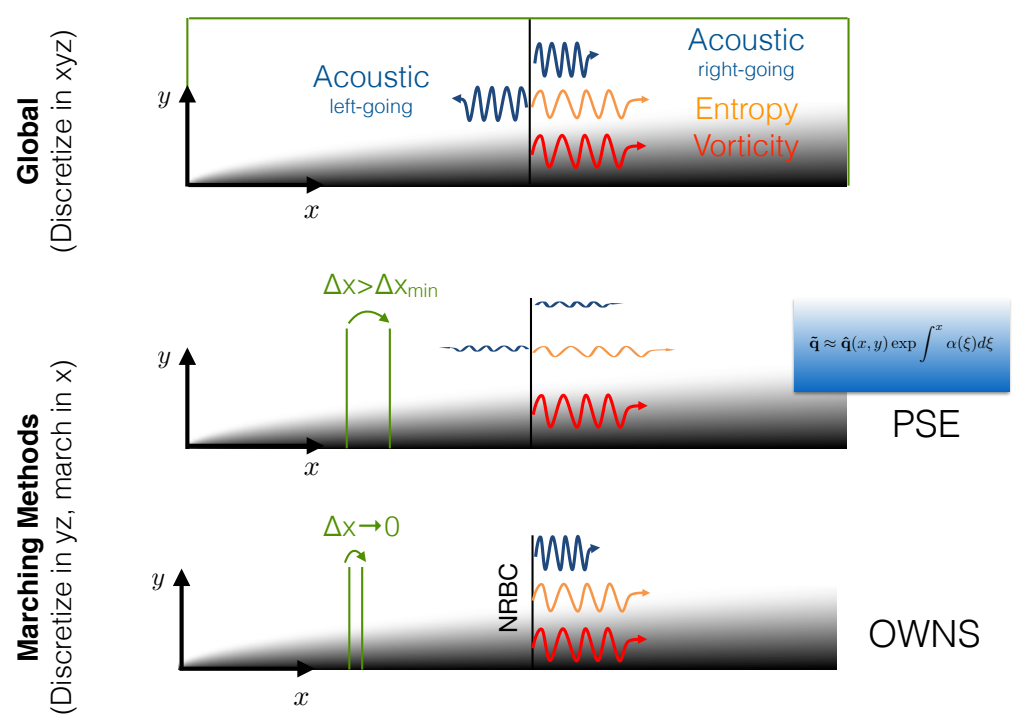

Fig. 1 Global versus marching methods PSE and OWNS on a flat-plate boundary layer.

In the present paper, we begin with the compressible Navier-Stokes equations and the resulting one-way equations in Sec. III] For brevity, we omit algorithmic details of optimal OWNS but the original formulation can be found in [18]. We validate optimal OWNS against global resolvent analyses from [19] and using our latest code CSTAT (Caltech Stability and Transition Analysis Toolkit) for a Mach 4.5 adiabatic flat-plate boundary layer in Sec. IV]. CSTAT can perform a variety of stability analyses from subsonic to hypersonic regimes for jets and boundary layers in non-orthogonal, body-fitted curvilinear coordinates with user-defined fluid properties. We demonstrate its capability by applying optimal OWNS on a Mach $638.1 \%$ scale model of the HIFiRE-5 elliptic cone [20] in Sec. V] 


\section{Methodology}

\section{A. Linearized equations of motion}

The non-dimensional compressible Navier-Stokes equations for an ideal gas are

$$
\begin{aligned}
& \frac{D \rho}{D t}+\rho(\nabla \cdot \mathbf{u})=0, \\
& \rho \frac{D \mathbf{u}}{D t}+\nabla p=\frac{1}{R e} \nabla \cdot \tau, \\
& \rho \gamma R \frac{D T}{D t}-(\gamma-1) \frac{D p}{D t}=\frac{\gamma-1}{\operatorname{Pr} R e} \nabla \cdot(k \nabla T)+\frac{\gamma-1}{\operatorname{Re}}(\tau: \nabla \mathbf{u}), \\
& \tau=2 \mu S+\kappa(\nabla \cdot \mathbf{u}) I, \\
& S=\frac{1}{2}\left(\nabla \mathbf{u}+(\nabla \mathbf{u})^{T}-\frac{2}{3}(\nabla \cdot \mathbf{u}) I\right), \\
& p=\rho R T \\
& \operatorname{Re}=\frac{\rho_{\infty}^{\dagger} a_{\infty}^{\dagger} \delta_{0}^{\dagger}}{\mu_{\infty}^{\dagger}} \\
& \operatorname{Pr}=\frac{\mu_{\infty}^{\dagger} c_{p_{\infty}}^{\dagger}}{k_{\infty}^{\dagger}} .
\end{aligned}
$$

We follow the two-step transformation from the Cartesian coordinate system to the physical non-orthogonal curvilinear coordinate system outlined in [21] and linearize all equations about a time-independent base flow such that

$$
q(\xi, \eta, \zeta, t)=\bar{q}(\xi, \eta, \zeta)+q^{\prime}(\xi, \eta, \zeta, t)
$$

where $q=[\rho, u, v, w, T]^{T}$ is the state vector. Due to algebraic complexity, we have automated this transformation using the Mathematica software and present the general matrix form of the resulting forced linear equation

$$
\begin{gathered}
G \frac{\partial q^{\prime}}{\partial t}+A_{\xi, i v s} \frac{\partial q^{\prime}}{\partial \xi}+A_{\eta, i v s} \frac{\partial q^{\prime}}{\partial \eta}+A_{\zeta, i v s} \frac{\partial q^{\prime}}{\partial \zeta}+A_{i v s} q^{\prime}=A_{\xi, v i s} \frac{\partial q^{\prime}}{\partial \xi}+A_{\eta, v i s} \frac{\partial q^{\prime}}{\partial \eta}+A_{\zeta, v i s} \frac{\partial q^{\prime}}{\partial \zeta}+ \\
A_{v i s} q^{\prime}+B_{\xi \xi} \frac{\partial^{2} q^{\prime}}{\partial \xi^{2}}+B_{\eta \eta} \frac{\partial^{2} q^{\prime}}{\partial \eta^{2}}+B_{\zeta \zeta} \frac{\partial^{2} q^{\prime}}{\partial \zeta^{2}}+B_{\xi \eta} \frac{\partial^{2} q^{\prime}}{\partial \xi \partial \eta}+B_{\xi \zeta} \frac{\partial^{2} q^{\prime}}{\partial \xi \partial \zeta}+B_{\eta \zeta} \frac{\partial^{2} q^{\prime}}{\partial \eta \partial \zeta}+G \mathcal{B}_{p} f_{p},
\end{gathered}
$$

where $f_{p}$ is a general primitive forcing term. After discretizing in the $\eta$ and $\zeta$ directions (if performing fully 3D analysis, otherwise we invoke spanwise periodicity and consider one wavenumber at a time), we map the resulting semi-discretized equation into characteristic space via the transformation

$$
\phi(\xi, \eta, \zeta, t)=T_{\phi}(\xi, \eta, \zeta) q^{\prime}(\xi, \eta, \zeta, t), \quad \widetilde{A}_{\xi, i v s}=T_{\phi} G^{-1} A_{\xi, i v s} T_{\phi}^{-1},
$$

where the rows of $T_{\phi}$ are the left eigenvectors of $G^{-1} A_{\xi, i v s}$, yielding

$$
\frac{d \phi}{d \xi}-\widetilde{M} \phi=m_{c}, \quad \phi\left(\xi=\xi_{0}, \eta, \zeta\right)=\phi_{0} .
$$

Eq. 5 is still exact, but cannot be solved as an initial value problem (IVP) in $\xi$ because $M$ has eigenvalues of both signs. In PSE, this equation is regularized to damp the upstream modes, whereas in OWNS the equation is parabolized by filtering out the modes with upstream group velocity [16, 22].

\section{Mach 4.5 Adiabatic Flat-Plate Validation Results}

\section{A. Optimal gain curves}

The normalized gain curves computed from OWNS are plotted against singular values from the global resolvent analyses of [19] in Fig. 2. Note that the absolute value of the gains has no physical meaning but the trend observed 
in the $\omega-\beta$ space allows resonance and pseudo-resonance of the flow response to be analyzed for a given harmonic forcing [19]. For all three instability types, we observe excellent agreement of the optimal $\omega$ and/or $\beta$, but with much reduced computational effort in the OWNS formulation.
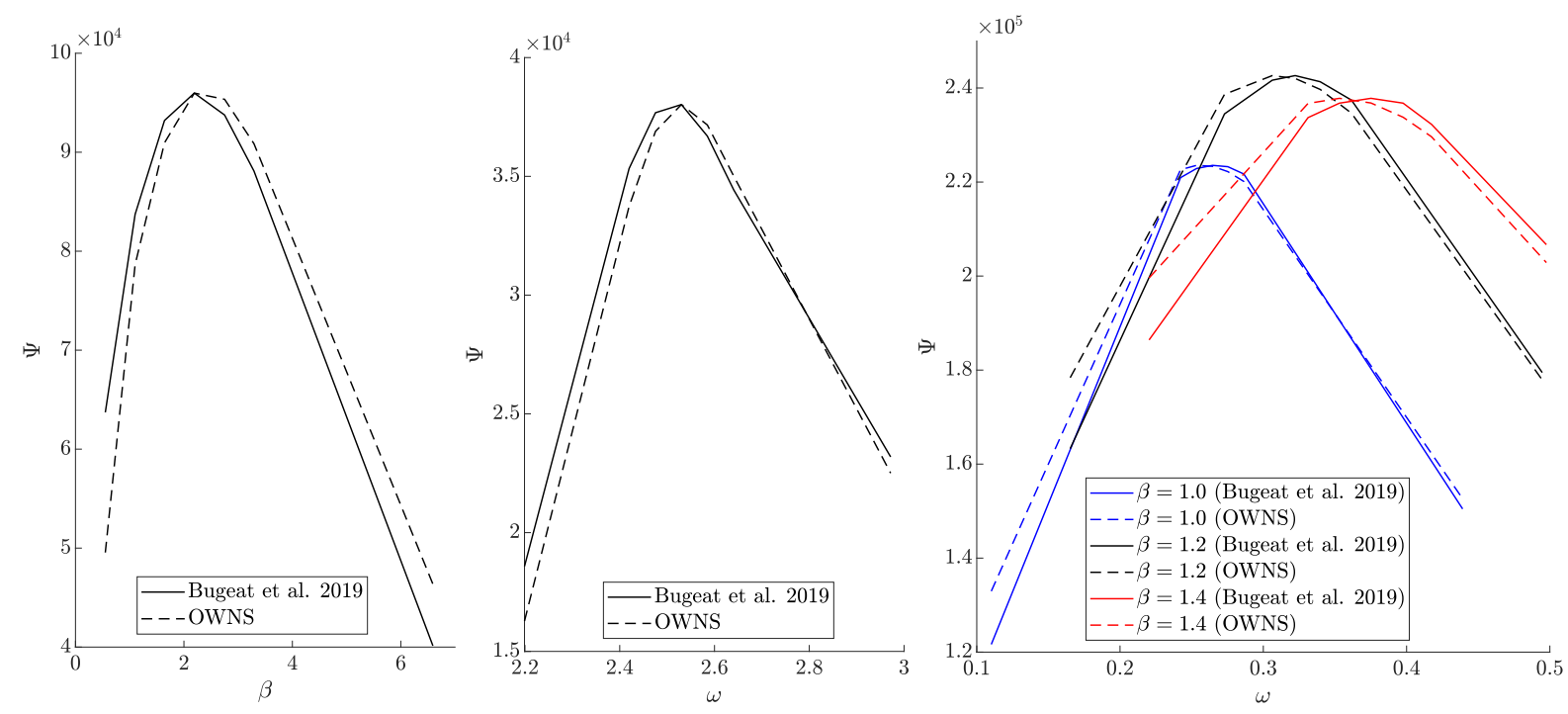

Fig. 2 Normalized gain curves for streaks (left), second mode (middle), and oblique first mode (right) between OWNS and [19] for Mach 4.5 adiabatic flat-plate boundary layer.

\section{B. Optimal forcing and response}

In this section, we compare the optimal forcing and responses between OWNS and global resolvent calculated by CSTAT using the same conditions from [19]. Table 1 summarizes the frequency and/or spanwise wavenumber used for each type of instability (corresponds to the optimal conditions from [19]). Overall, Figs. 3 and 4 show agreement between the OWNS and global results. Note that since only the real component is plotted, the magnitude and/or polarity differences is due to the phase shift between the two methods (this phase is arbitrary). Furthermore, the streamwise domain for global resolvent is bounded by inlet and outlet sponges to model open boundaries. The presence of the inlet sponge forces the forcing to effectively begin further downstream, yielding a delayed response. This demonstrates an additional advantage of using OWNS to compute the optimal perturbations and responses.

Table 1 Optimal $F$ and/or $\beta^{\dagger}$ used for streaks, oblique first mode, and second mode from [19].

\begin{tabular}{ccc}
\hline & $\beta^{\dagger}[1 / \mathrm{m}]$ & $F$ \\
\hline Streaks & $2.0534 \times 10^{4}$ & $1.7832 \times 10^{-7}$ \\
First mode & $1.1200 \times 10^{4}$ & $2.8531 \times 10^{-5}$ \\
Second mode & 0 & $2.2290 \times 10^{-4}$ \\
\hline
\end{tabular}

For streaks, the optimal forcing consists of streamwise counter-rotating vortices that lift the streamwise base flow momentum. This is referred to as the lift-up mechanism and yields a response that contains primarily streaks of highly amplified streamwise velocity stretching in the streamwise direction. For the oblique first mode, the optimal forcing field contains upstream-titled structures that is emblematic of the non-modal Orr mechanism. This generates an oblique wave response with relatively large streamwise velocity. Lastly, for the second mode, we see the classical trapped acoustic waves between the wall and relative sonic line as well as thermodynamic amplification near the generalized inflection point in the response fields (two coexisting mechanisms). For such a response, we require the optimal forcing to be concentrated near the generalized inflection point.

The contour plots of the suboptimal forcing and responses between OWNS and global resolvent are shown in Figs. 5 and 6 , respectively. We again observe agreement between the two methods, where we see an increased number of 
wavepackets in the forcing and responses due to the orthogonality between optimal and suboptimal modes.
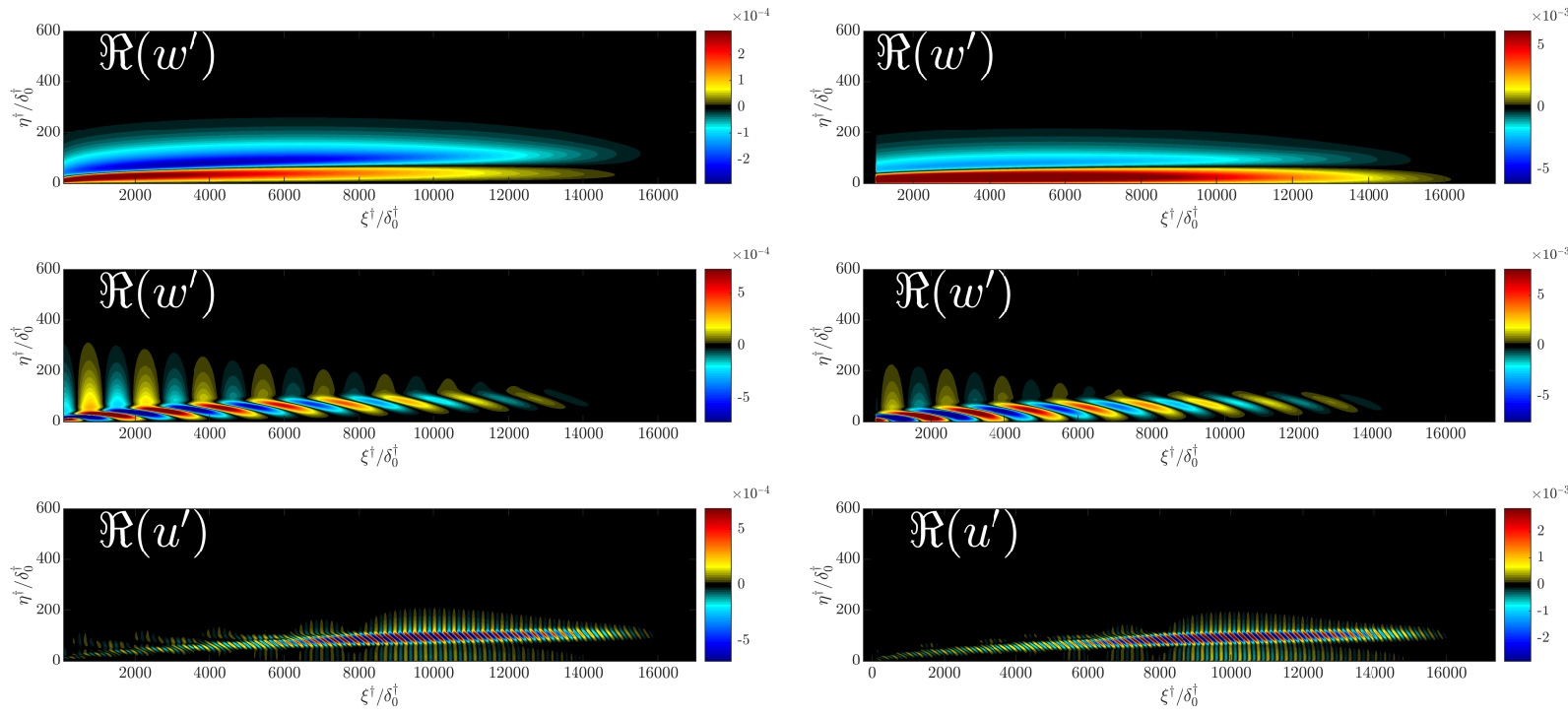

Fig. 3 Comparison of optimal forcing fields for streaks (top), oblique first mode (middle), and second mode (bottom) for adiabatic Mach 4.5 flat-plate boundary layer between OWNS (left) and global resolvent (right).
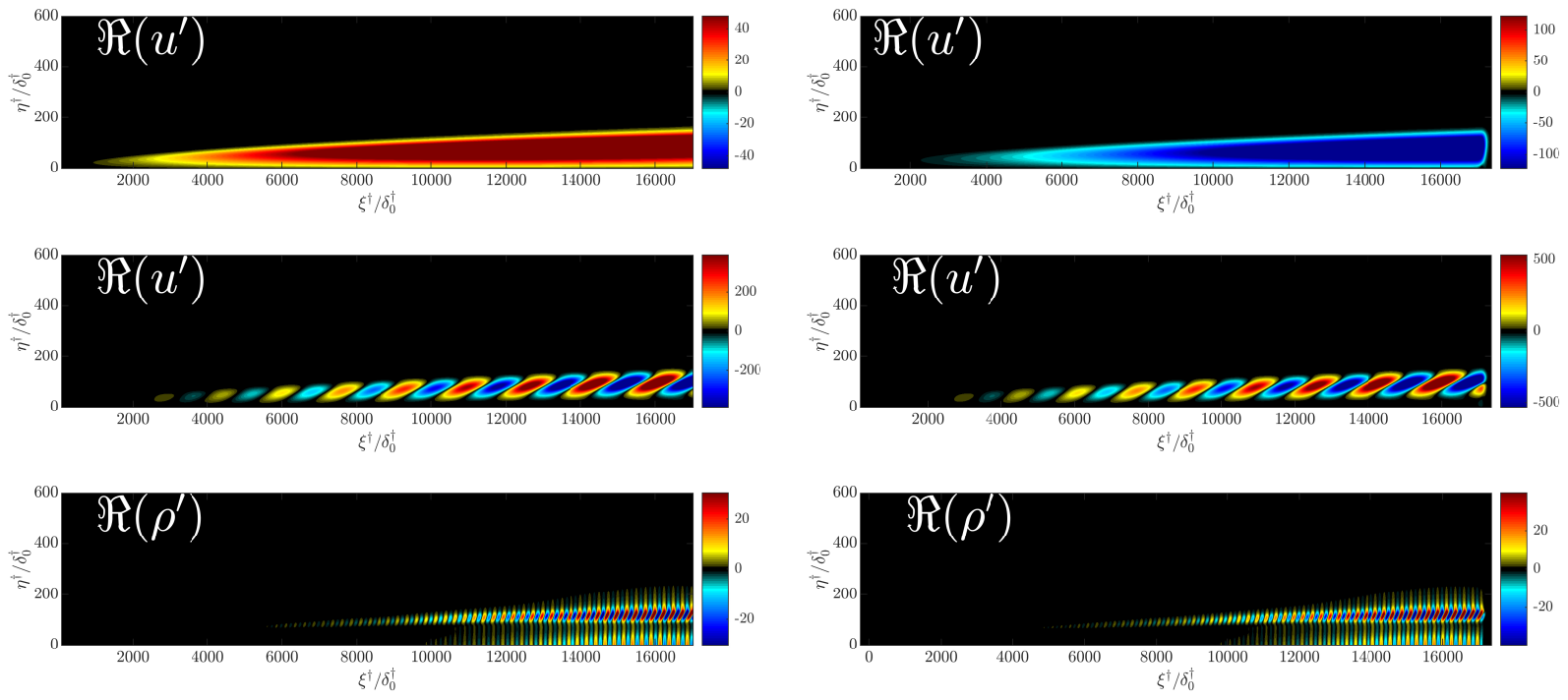

Fig. 4 Comparison of optimal response fields for streaks (top), oblique first mode (middle), and second mode (bottom) for adiabatic Mach 4.5 flat-plate boundary layer between OWNS (left) and global resolvent (right). 

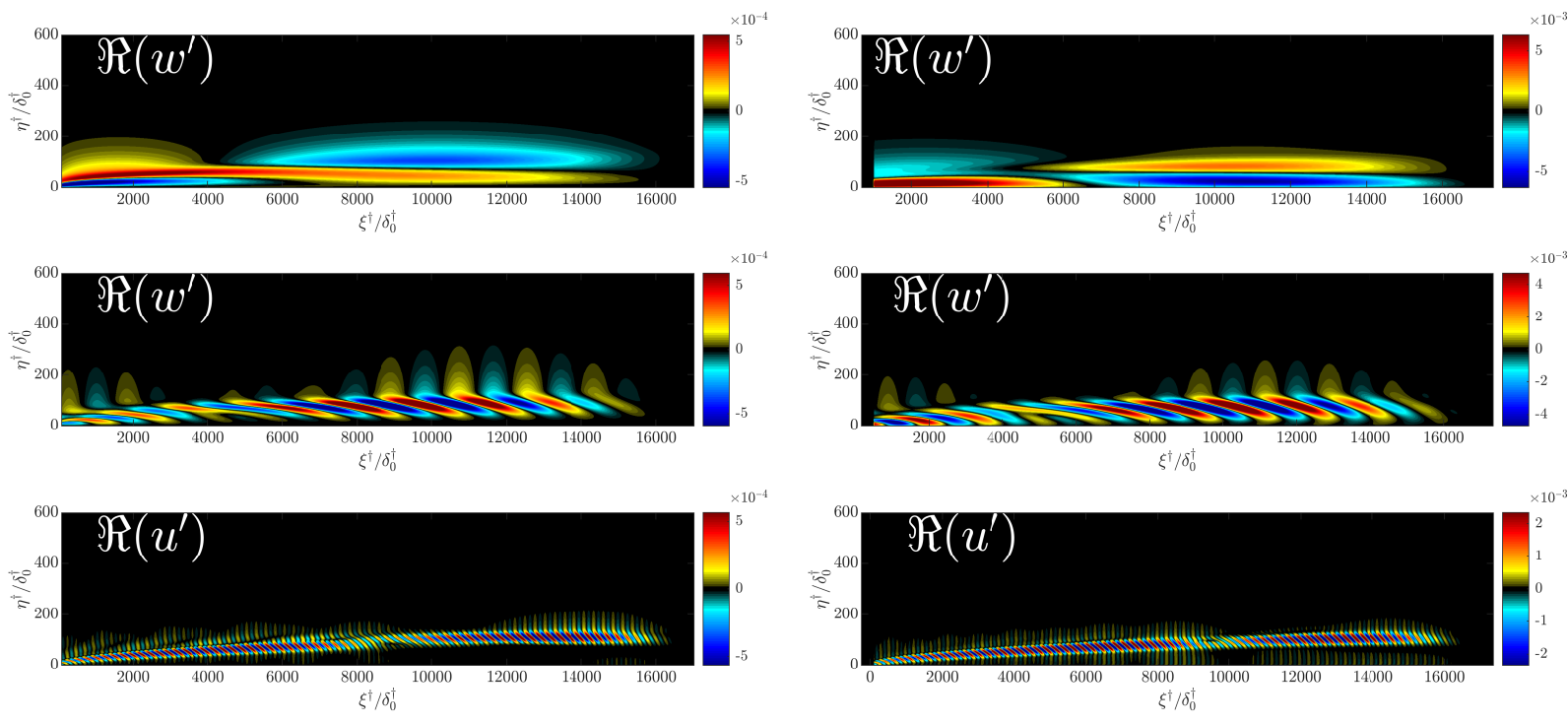

Fig. 5 Comparison of suboptimal forcing fields for streaks (top), oblique first mode (middle), and second mode (bottom) for adiabatic Mach 4.5 flat-plate boundary layer between OWNS (left) and global resolvent (right).
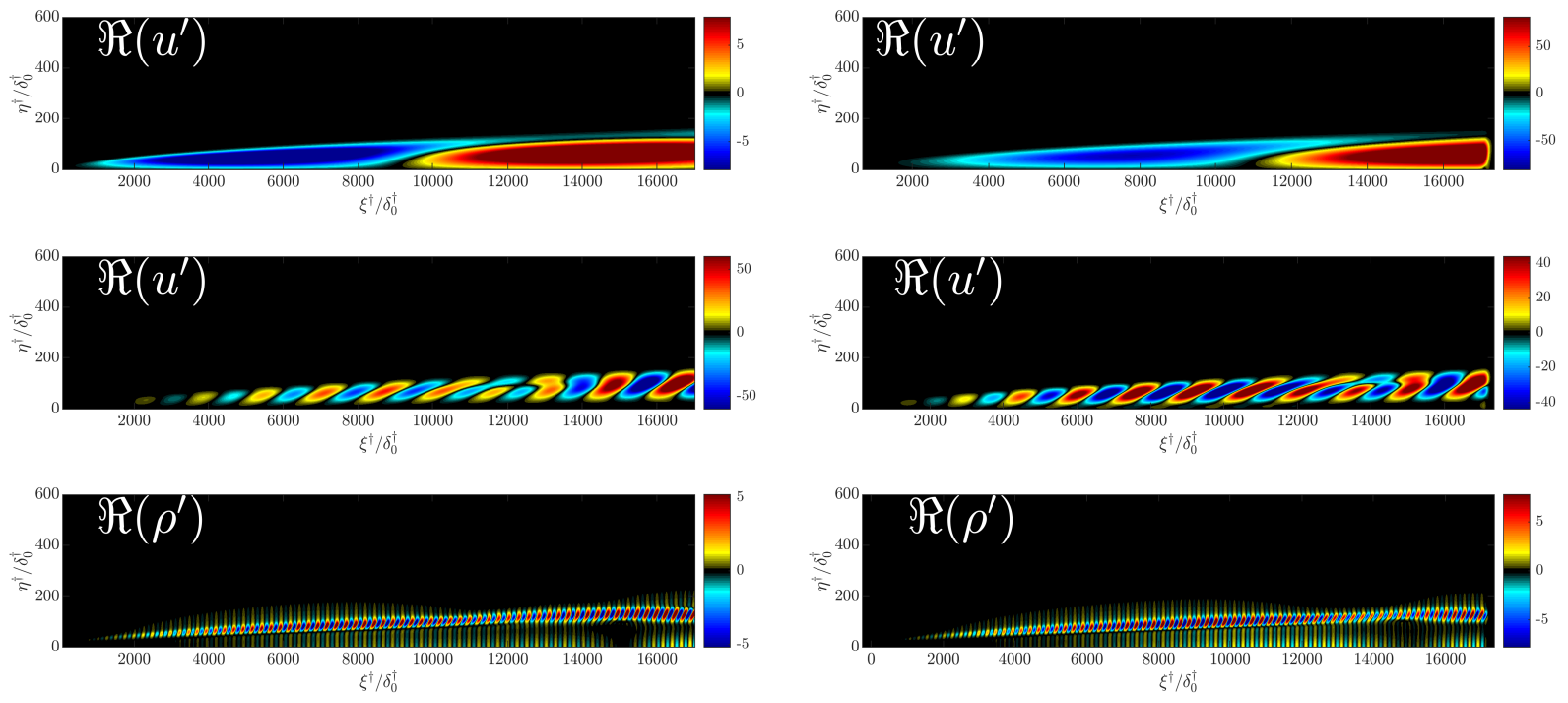

Fig. 6 Comparison of suboptimal response fields for streaks (top), oblique first mode (middle), and second mode (bottom) for adiabatic Mach 4.5 flat-plate boundary layer between OWNS (left) and global resolvent (right).

\section{Mach 6.0 HIFiRE-5 Results}

To assess the optimal OWNS framework for more complex geometries, we perform a preliminary 2D $(\beta=0)$ optimization along the centerline of the HIFiRE-5 elliptic cone at $f^{\dagger}=80 \mathrm{kHz}$ (this frequency was shown to have the highest LST N-factor from [23]). We include streamwise curvature with no input or output restrictions in the optimal calculations. The forcing fields in Fig. 7) show some resemblance to the second mode from the Mach 4.5 flat-plate case, but the responses contain complex, non-canonical second mode structures. This may be attributed to the primary vortex roll-up near the leeward line inducing strong spanwise variations [24], thereby suggesting a fully 3D analysis may be required to accurately characterize the optimal disturbances along the centerline. Lastly, the suboptimal forcing and 
responses in Fig. 8 show increased number of wavepackets due to optimal-suboptimal modal orthogonality, as seen in the Mach 4.5 flat-plate case.
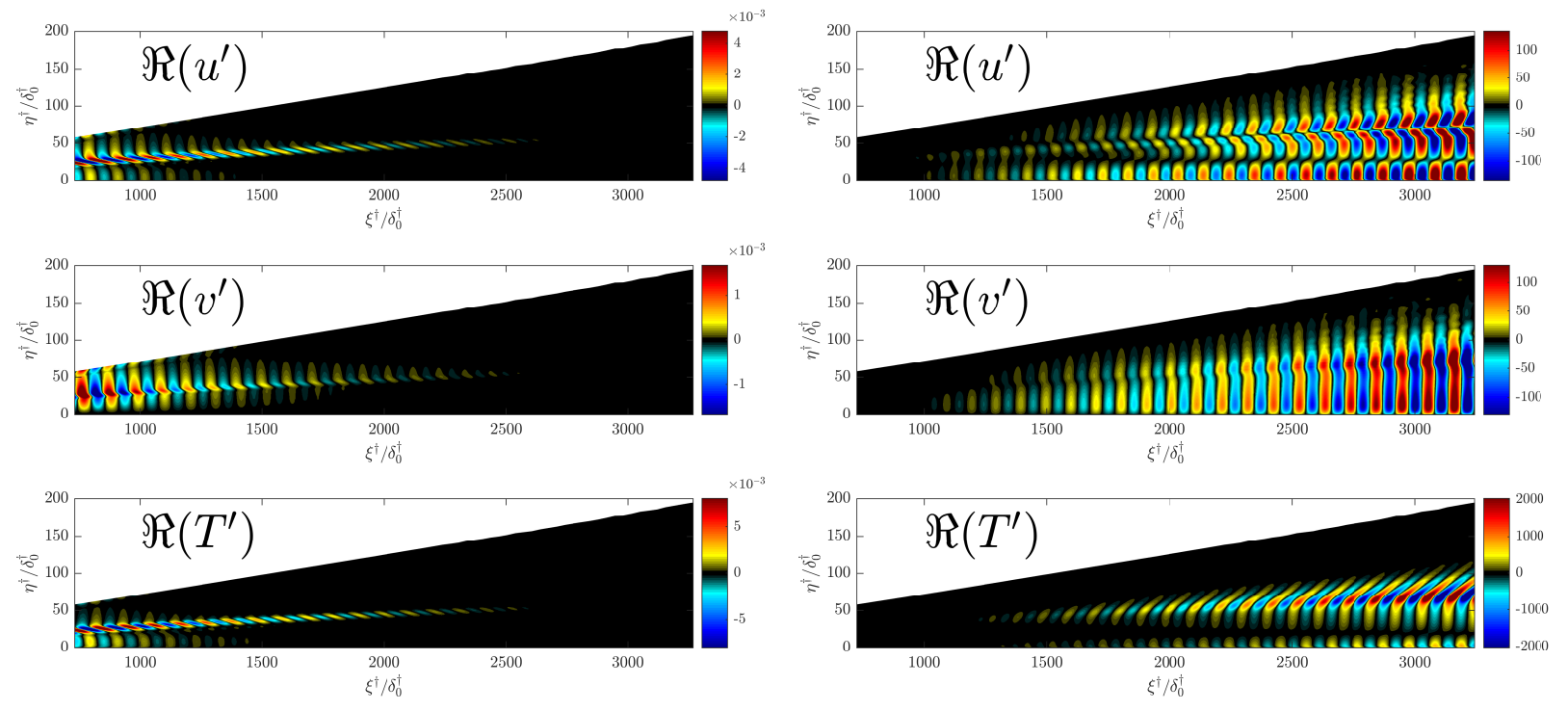

Fig. 7 Optimal OWNS forcing (left) and response (right) fields at $f^{\dagger}=80 \mathrm{kHz}$ for Mach 6.0 HIFiRE-5 elliptic cone.
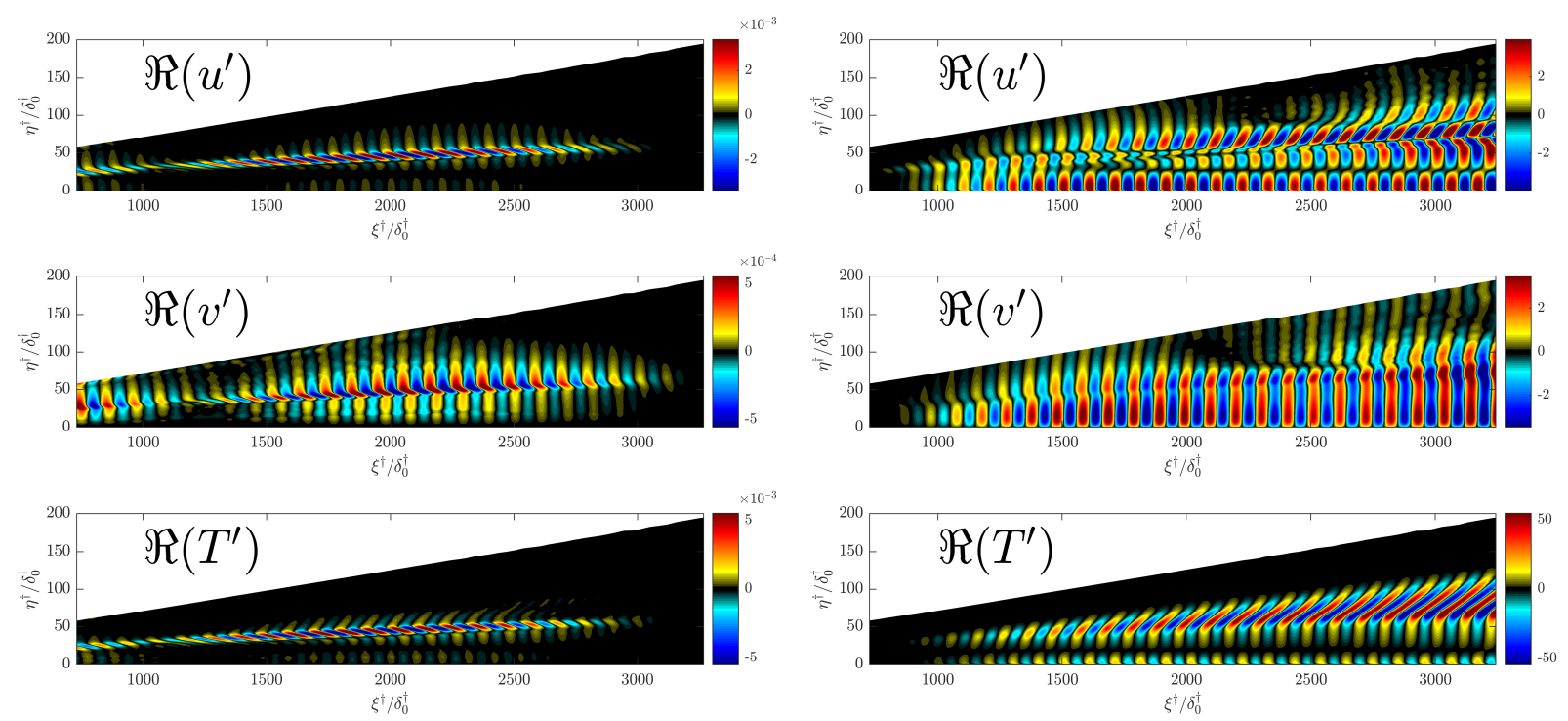

Fig. 8 Suboptimal OWNS forcing (left) and response (right) fields at $f^{\dagger}=80 \mathrm{kHz}$ for Mach 6.0 HIFiRE-5 elliptic cone.

\section{Conclusions and Future Work}

Using OWNS, we have computed optimal and suboptimal, modal and non-modal, linear perturbations and forced responses of a Mach 4.5 flat plate and Mach $6.0 \mathrm{HIFiRE}-5$ elliptic cone. The flat-plate case was first validated against full global calculations from the literature [19]. Our computations show that OWNS can accurately and efficiently determine the worst-case disturbances. The flat-plate case illuminated various fundamental mechanisms present for optimal transition and the HIFiRE-5 analyses demonstrated the applicability of optimal OWNS for engineering design. 
In the near future, we will expand our optimal disturbance calculation to consider further geometries including sharp cones and fully inhomogeneous flows (via planar OWNS marching) such as HIFiRE-5 and BOLT [25]. We will also examine the receptivity problem for the geometries in detail by prescribing different cost functions that characterize the worst-case disturbances.

\section{Acknowledgments}

This work has been supported by The Boeing Company through the Strategic Research and Development Relationship Agreement CT-BA-GTA-1, and more recently through the Office of Naval Research via grant N00014-21-1-2158. We also acknowledge support of the Natural Sciences and Engineering Research Council of Canada via the Postgraduate Doctoral Scholarship (PGSD3-532522-2019).

\section{References}

[1] Hirschel, E. H., Laminar-Turbulent Transition and Turbulence in High-Speed Viscous Flow, chapter and pages, pp. $263-310$.

[2] Fedorov, A., "Transition and Stability of High-Speed Boundary Layers," Annual Review of Fluid Mechanics, Vol. 43, No. 1, 2011, p. 79-95.

[3] Mack, L. M., "Special course on stability and transition of laminar flow," AGARD report, Vol. 709, 1984.

[4] Mack, L., “Boundary-Layer Stability Theory. Part B., Jet Propulsion Lab., Pasadena, CA,” Document 900-277, Rev. A, 1969.

[5] Saric, W. S., “Görtler Vortices,” Annual Review of Fluid Mechanics, Vol. 26, No. 1, 1994, p. 379-409.

[6] Knisely, C. P., and Zhong, X., "Sound radiation by supersonic unstable modes in hypersonic blunt cone boundary layers. II. Direct numerical simulation," Physics of Fluids, Vol. 31, No. 2, 2019, p. 024104.

[7] Arnal, D., and Casalis, G., "Laminar-turbulent transition prediction in three-dimensional flows," Progress in Aerospace Sciences, Vol. 36, No. 2, 2000, p. 173-191.

[8] Saric, W. S., Reed, H. L., and White, E. B., "Stability and Transition of Three-Dimensional Boundary Layers," Annual Review of Fluid Mechanics, Vol. 35, No. 1, 2003, p. 413-440.

[9] Kuehl, J., and Paredes, P., “Gortler Modified Mack-modes on a Hypersonic Flared Cone," 54th AIAA Aerospace Sciences Meeting, 2016.

[10] Ma, Y., and Zhong, X., "Receptivity of a supersonic boundary layer over a flat plate. Part 1. Wave structures and interactions," Journal of Fluid Mechanics, Vol. 488, 2003, p. 31-78.

[11] Towne, A., Rigas, G., and Colonius, T., "A critical assessment of the parabolized stability equations," Theoretical and Computational Fluid Dynamics, Vol. 33, No. 3-4, 2019, p. 359-382.

[12] Nichols, J. W., and Lele, S. K., "Global modes and transient response of a cold supersonic jet," Journal of Fluid Mechanics, Vol. 669, 2011, pp. 225-241.

[13] Monokrousos, A., Åkervik, E., Brandt, L., and Henningson, D. S., "Global three-dimensional optimal disturbances in the Blasius boundary-layer flow using time-steppers,” J. Fluid Mech., Vol. 650, 2010, pp. 181-214.

[14] Nichols, J. W., and Candler, G. V., "Input-output analysis of complex hypersonic boundary layers," AIAA Scitech 2019 Forum, 2019, p. 1383.

[15] Cook, D. A., Knutson, A., Nichols, J. W., and Candler, G. V., "Matrix methods for input-output analysis of 2D and 3D hypersonic flows," AIAA Scitech 2020 Forum, 2020, p. 1820.

[16] Towne, A., and Colonius, T., "One-way spatial integration of hyperbolic equations," Journal of Computational Physics, Vol. 300,2015 , p. 844-861.

[17] Rigas, G., Colonius, T., and Beyar, M., "Stability of wall-bounded fows using one-way spatial integration of Navier-Stokes equations," 55th AIAA Aerospace Sciences Meeting, 2017.

[18] Rigas, G., Schmidt, O. T., Colonius, T., and Brès, G. A., "One Way Navier-Stokes and resolvent analysis for modeling coherent structures in a supersonic turbulent jet," 23rd AIAA/CEAS Aeroacoustics Conference, 2017. 
[19] Bugeat, B., Chassaing, J.-C., Robinet, J.-C., and Sagaut, P., "3D global optimal forcing and response of the supersonic boundary layer," Journal of Computational Physics, Vol. 398, 2019, p. 108888.

[20] Kimmel, R., Adamczak, D., Berger, K., and Choudhari, M., "HIFiRE-5 Flight Vehicle Design," 40th Fluid Dynamics Conference and Exhibit, 2010.

[21] Demirdzic, I., Gosman, A., Issa, R., and Peric, M., "A calculation procedure for turbulent flow in complex geometries," Computers \& Fluids, Vol. 15, No. 3, 1987, p. 251-273.

[22] Towne, A., "Advancements in jet turbulence and noise modeling: accurate one-way solutions and empirical evaluation of the nonlinear forcing of wavepackets," Ph.D. thesis, California Institute of Technology, 2016.

[23] Kamal, O., Rigas, G., Lakebrink, M. T., and Colonius, T., "Application of the One-Way Navier-Stokes (OWNS) Equations to Hypersonic Boundary Layers,” AIAA Aviation 2020 Forum, 2020.

[24] Li, F., Choudhari, M., Chang, C.-L., White, J., Kimmel, R., Adamczak, D., Borg, M., Stanfield, S., and Smith, M., "Stability Analysis for HIFiRE Experiments," 42nd AIAA Fluid Dynamics Conference and Exhibit, 2012.

[25] Thome, J., Dwivedi, A., Nichols, J. W., and Candler, G. V., "Direct numerical simulation of BOLT hypersonic flight vehicle," 2018 Fluid Dynamics Conference, 2018. 